\title{
TRAVELING WAVES ON ELECTRIC POWER SYSTEMS
}

\author{
L. V. BEWLEY
}

The problems of traveling waves on the transmission lines of a power system ${ }^{1}$ differ considerably from those of traveling waves on telephone or telegraph circuits. The primary object in the case of the former is to know how to protect the system from abnormal voltage disturbances which might damage apparatus or cause discontinuity of service; whereas the object in the case of the latter is the transmission of signals. Attenuation, distortion, wave shape modification, and successive reflections are deliberately sought after on the power system as a means of rendering the surges innocuous, but these effects must be carefully avoided or nullified on the communication circuits so as to preserve the wave shape and transmit the signal with strength, fidelity, and without interference. On the power lines, the surges often originate from unknown causes, or at the point of origin are of unknown magnitude and shape (except from a statistical point of view); while on the communication circuits the initial shape and magnitude of the wave train are known with exactness. External fields (due to charged clouds), corona, flashovers, faults, and so on are of great importance with respect to surges; but are of no concern in the normal functioning of a telephone or telegraph line. Thus on the power lines surges originate from external or undesirable causes and every effort must be made to withstand or control them; while on communication circuits the transients are the direct means to the end.

These differences have led to corresponding differences in the mathematical approach. The power engineer is satisfied with approximations which would be intolerable to the communications engineer, and he is willing to take a license with mathematical rigor which would make any self-respecting mathematician groan. Higher mathematics has found little or no application in the study of surges on power systems. This has been due primarily to the fact that the boundary conditions are not definite enough to justify purely mathematical refinements; particularly since engineering results must be obtained in a short time by men who are not mathematicians. However, there are numerous aspects of the problem which lend themselves to mathematical excursions. It is my purpose in this lecture to

An address delivered before the meeting of the Society in Bethlehem, Pa., on December 30, 1941, by invitation of the Program Committee under the title The mathematical theory of traveling waves; received by the editors January 10, 1942.

1 Traveling Waves on Transmission Systems, L. V. Bewley, New York, Wiley. 
give you the engineering methods, and perhaps, here and there, to suggest how advanced mathematics might be applied.

The general problem. Figure 1 indicates a multi-conductor transmission line, protected by a ground wire ${ }^{2}$ at the peak of the towers, terminating at a power station containing a lightning arrester, a circuit breaker, a transformer, and a generator. A cloud, floating over the line, has been charged by the action of the rising air currents on

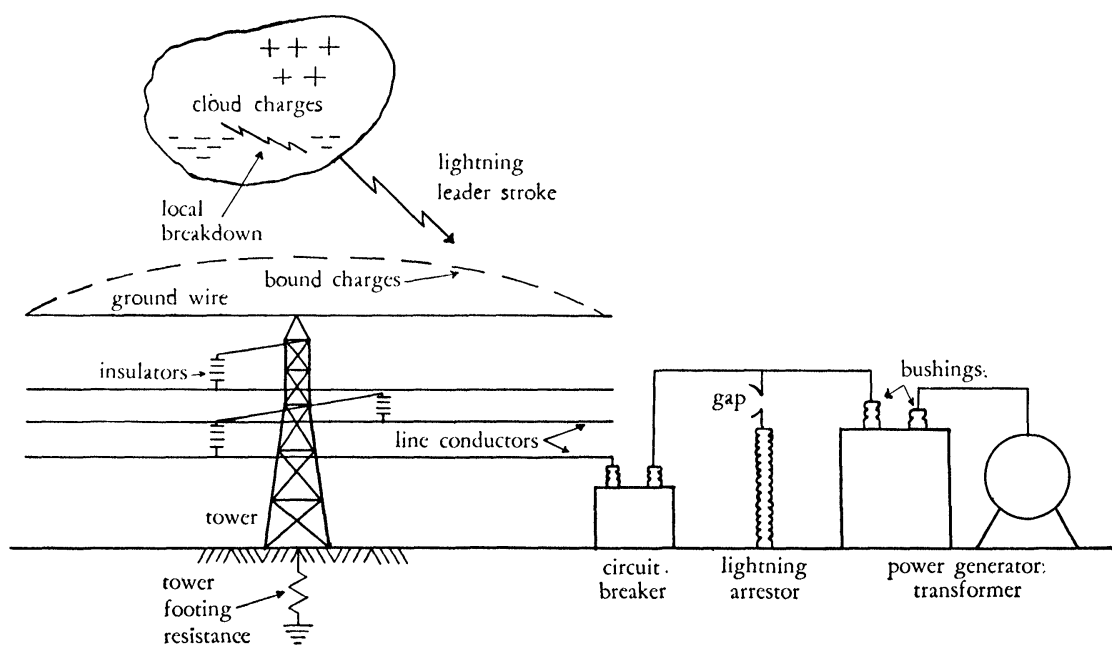

FIG. 1

the falling raindrops, and this cloud charge has induced a stationary counter-charge on the conductors of the transmission line. As the potential of the cloud increases, local breakdowns occur throughout its mass, thereby uniting some of its regions and making available, through partially ionized paths, a reservoir of charge. Eventually the field gradient reaches an intensity sufficient to initiate a leader stroke or dart, which starts towards ground. The progress of this dart is not continuous, but by jerks, each jerk depending upon the supply of additional charge to the head of the dart. It is like the armored force in a blitzkrieg break-through-it strikes to the limit of its capabilities and must then wait for tactical reinforcements and logistical support before renewing its attack. As the dart approaches earth, the field

2 The purpose of a ground wire is to intercept a lightning stroke and thereby protect the power conductors from destructive surges. Those surges which do come in to the station are absorbed by the lightning arrestor. 
gradient at the transmission line increases and this causes a migration of charge up through the towers onto the ground wire, and from the remote parts on the line conductors towards the region of field concentration. The dart finally makes contact-say with the ground wire at the tower - and a lightning surge moves out in both directions on the ground wire, inducing waves on the line conductors. But when these waves reach the next tower, reflections occur, and very soon all the neighboring spans are filled by numerous waves reflecting back and forth, and perhaps flashovers have taken place to the line conductors. These waves are rushing, with the speed of light, towards the power station, where they may enter the windings of transformers and generators, causing steep gradients which may breakdown the turn-to-turn insulation, and oscillations which may develop destructive voltages on the major insulation to ground. Perhaps a bushing flashover, or an insulation failure will cause the circuit-breaker to function, interrupting the normal 60 cycle power current, and this operation will initiate a new transient which is called a "switching surge." And perhaps an insulator flashover out on the line will culminate in intermittent arcing which may result in a cumulative building-up of dangerous voltages - the so-called "arcing ground."

The engineer does not tackle this problem in its entirety by attempting to include all the terminal apparatus as boundary conditions. Rather is he compelled to make a piecemeal attack, by handling each part of the problem as a separate and independent proposition, thereby isolating and defeating them in detail. To this end, certain aspects of the problem will be considered under four main headings.

Multi-velocity waves (tensor notation). Consider a system of $n$ overhead transmission line conductors with voltages $e_{r}$ currents $i^{r}$, charges $Q^{r}$, and fluxes $\phi_{r}$. Then in terms of Maxwell's electrostatic potential coefficients $p_{r s}$

$$
e_{r}=p_{r s} Q^{s}
$$

from which

$$
Q^{r}=K^{r s} e_{s}
$$

where $K^{r s}$ is the inverse of $p_{r s}$ in the matrix [ $\left.p_{r s}\right]$. These coefficients are calculated for parallel cylindrical conductors in the presence of ground by including the images of the conductors in the ground surface. 
The magnetic flux linkages are given in terms of the inductance coefficients by

$$
\phi_{r}=L_{r s} i^{s}
$$

There are leakage currents flowing to ground and between conductors of amount $G^{r s} e_{s}$. The $G^{r s}$ coefficients are supposed to include the effects of both leakage and corona.

And finally, there are resistance drops $R_{r s} i^{s}$ in the conductors due to the flow of currents.

Herefrom, the differential equations for the multi-conductor transmission system become (putting $p=\partial / \partial t$, in the Heaviside sense)

$$
\begin{aligned}
& -\frac{\partial e_{r}}{\partial x}=p \phi_{r}+R_{r t} i^{t}=\left(L_{r t} p+R_{r t}\right) i^{t}, \\
& -\frac{\partial i^{t}}{\partial x}=p Q^{t}+G^{t s} e_{s}=\left(K^{t s} p+G^{t s}\right) e_{s} .
\end{aligned}
$$

Eliminating $i^{t}$ there results

$$
\left(J_{r}^{s}-\delta_{r}^{s} \frac{\partial^{2}}{\partial x^{2}}\right) e_{s}=0
$$

in which $\delta_{r}^{s}$ is the Kronecker delta and

$$
J_{r}^{s}=\left(L_{r t} p+R_{r t}\right)\left(K^{t s} p+G^{t s}\right) \text {. }
$$

Now if the losses are ignored $\left(R_{r s}=0, G^{r s}=0\right),(6)$ is satisfied by the traveling wave ${ }^{3}$

$$
e_{8}=a_{s}^{(\alpha)} f_{(\alpha)}\left(x-v_{(\alpha)} t\right)
$$

which substituted in (6) gives

$$
\left(L_{r t} K^{t s} p^{2}-\delta_{r}^{s} \frac{\partial^{2}}{\partial x^{2}}\right) a_{s}^{(\alpha)} f_{(\alpha)}\left(x-v_{(\alpha)} t\right)=0 .
$$

Since this equation must be satisfied for waves of the same velocity

$$
\left(L_{r t} K_{v_{(\alpha)}^{t}}^{2}-\delta_{r}^{\delta}\right) a_{s}^{(\alpha)}=0 .
$$

The velocities are given by the roots of the determinant

\footnotetext{
${ }^{3}$ An enclosed index is used here to suspend the summation convention.
} 


$$
\left|L_{r t} K_{v_{(\alpha)} t_{(\alpha)}^{2}}-\delta_{r}^{s}\right|=\left|c_{r}^{. s}\right|=0 .
$$

To each root of (11) there correspond $n$ values of $a_{s}^{(\alpha)}$, and any $(n-1)$ of them may be determined in terms of one value taken arbitrarily. Let this one value be $a_{1}^{(\alpha)}=1$. Solving (10)

$$
a_{s}^{(\alpha)}=\frac{-A_{s}^{r}}{\left|b_{r}^{\cdot s}\right|}\left(L_{r t} K_{v_{(\alpha)}^{t 1}}^{2}\right) \quad \text { where } s \neq 1, r \neq 1,
$$

in which $\left|b_{r}^{s}\right|$ is $\left|c_{r}^{s}\right|$ with the $s=1$ row and $r=1$ column deleted, and $A_{s}^{r}$ is the cofactor of $b_{r}^{s}$ in $\left|b_{r}^{s}\right|$.

The complete solution then becomes

$$
e_{r}=a_{r}^{\alpha}\left[f_{\alpha}\left(x-v_{(\alpha)} t\right)+F_{\alpha}\left(x+v_{(\alpha)} t\right)\right] .
$$

By (5) the corresponding currents are

$$
i^{r}=-p \int K^{r s} e_{s} d x=K^{r s} a_{s}^{\alpha}\left(f_{\alpha}-F_{\alpha}\right) v_{(\alpha)}=Y^{r \alpha}\left(f_{\alpha}-F_{\alpha}\right) .
$$

Transition points. Consider the general case of Figure 2 in which any number of incoming lines terminate at a transition point consisting of an inter-connected network and any number of outgoing

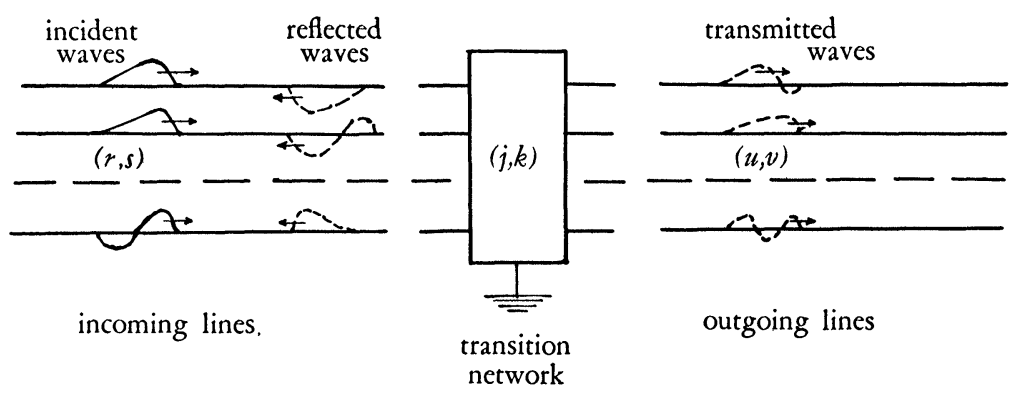

FIG. 2

lines. When the incident waves on the incoming lines reach the transition point, currents will flow into the network, transmitted waves will move out on the outgoing lines, and reflected waves will start back on the incoming lines. In tensor notation let:

$z_{r s}=$ surge impedances of incoming lines, ${ }^{4}$

${ }^{4}$ The surge impedance of a line is the coefficient of proportionality between its voltage and current $(e=z i)$. Its reciprocal is called surge admittance. For surges on transmission lines, these parameters are essentially constant, and are an indication of the current associated with a surge of given voltage. 
$y^{r s}=$ surge admittances of incoming lines (inverse of $z^{r s}$ ),

$z_{u v}=$ surge impedances of outgoing lines,

$y^{u v}=$ surge impedances of outgoing lines (inverse of $z_{u v}$ ),

$Z_{j k}=$ branch impedances of the network,

$C_{\alpha^{\prime}}^{\alpha}=$ transformation tensor specifying the total interconnections of the network and outgoing lines.

Then

(1) $Z_{\alpha \beta}=\left(Z_{j k}+z_{u v}\right)=$ impedance of network and outgoing lines before interconnection,

(2) $Z_{\alpha^{\prime} \beta^{\prime}}=C_{\alpha^{\prime}}^{\alpha} C_{\beta^{\prime}}^{\beta} Z_{\alpha \beta}=$ impedance after interconnection of the network and outgoing lines.

Now $Z_{\alpha^{\prime} \beta^{\prime}}$ may include branches other than those connected to the incoming lines. The open circuit branches will have been eliminated by $C_{\alpha^{\prime}}^{\alpha}$, but the branches other than those connected to the incoming lines will have to be eliminated by the substitutions:

$$
\begin{aligned}
Z_{\alpha^{\prime} \beta^{\prime}} & =Z_{r s}+Z_{r q}+Z_{p s}+Z_{p q}, \\
E_{r} & =Z_{r s} I^{s}+Z_{r q} I^{q}, \\
0 & =Z_{p s} I^{s}+Z_{p q} I^{q},
\end{aligned}
$$

from which

$$
E_{r}=\left(Z_{r s}-Z_{r q} Y^{q r} Z_{p s}\right) I^{s}=Z_{r s}^{\prime} I^{s} .
$$

Now let $\left(e_{r}, i^{r}\right)$ and $\left(e^{\prime}{ }_{r}, i^{\prime r}\right)$ be the incident and reflected waves, respectively, on the incoming line. Then at the transition point

$$
\begin{aligned}
& e_{r}+e_{r}^{\prime}=E_{r}=Z_{r s}^{\prime} I^{s}, \\
& i^{r}+i^{\prime r}=I^{r},
\end{aligned}
$$

so that

$$
e_{r}+e_{r}^{\prime}=Z_{r s}^{\prime}\left(i^{r}+i^{\prime r}\right)=Z_{r s}^{\prime} y^{s t}\left(e_{t}-e_{t}^{\prime}\right)
$$

from which

$$
\left(\delta_{r}^{t}+Z_{r s}^{\prime} y^{s t}\right) e_{t}^{\prime}=\left(-\delta_{r}^{t}+Z_{r s}^{\prime} y^{s t}\right) e_{t} .
$$

This system of equations defines the reflected voltage waves $e_{r}^{\prime}$.

The total voltage at the transition point then follows by (7), the total current by (8), the remaining network currents by (5) and the network voltages by (4), and so on.

Successive reflections. The calculation of successive reflections is oftentimes a long and involved process; particularly in those cases 
where reflections may occur from a whole series of neighboring junctions. An example is the case of lightning striking the ground wire at midspan. The incident waves move out in opposite directions until they reach the nearest towers, where they reflect as a consequence of the surge impedance of the continuing ground wire in parallel with that of the tower. Transmitted waves quickly reach the foot of the tower from which they reflect as a result of the ground resistance. Other waves reflect from the next tower, and from the next after that, and so on. Thus within a few microseconds the system is alive with a whole series of waves moving in different directions, arriving at different times, of different magnitudes and polarity, and having experienced different attenuations and distortions.

In order to keep track of all these components a lattice diagram has been devised, such as shown in Figure 3 for the case of lightning striking a ground wire at midspan. The progress of each wave component is easily followed as it slides downhill along its zig-zag path, giving rise to reflections at each junction. Thus at any instant of time the waves at all points on the line can be identified; or at.any point on the line the time of arrival of each wave can be seen.

To construct such a reflection lattice it is first necessary to determined the reflection and refraction coefficients ${ }^{5}$ at each junction, and to post these on the sketch of the system being studied, as has been done on Figure 3. The coefficients are, in general, Heaviside operators, such that when operating on an incident wave (regarded as a time function reckoned from its instant of arrival at the particular junction) they give the reflected or transmitted wave. Those shown in Figure 3 are $A, B, C, D, B^{\prime}, C^{\prime}, D^{\prime}$. The initial wave coming down the lightning stroke of surge impedance $2 Z_{0}$ (the 2 is occasioned by the condition of symmetry which permits the amputation of the system to the left of the stroke) refracts onto the ground wire a wave $A \cdot f(t)$ which moves on to the top of Tower 1 , where it reflects a portion $A B^{\prime} \cdot f(t)$ back towards its origin, and transmits a portion $A B \cdot f(t)$ to the next ground wire section and also down the tower. When the wave reaches the foot of the tower it reflects a portion $A B D^{\prime} \cdot f(t)$ back up the tower. Likewise reflections return from the top and foot of Tower 2 and from the towers beyond. Now each of these reflections could be traced out independently on the lattice and all waves fully accounted for. But the labor is great. The work can be

"A "reflection coefficient" is the operator determined from equation (10) of the previous section on transition points, which permits the calculation of a reflected wave in terms of the incident wave. Likewise, the "refraction coefficient" gives the transmitted wave. 
simplified by introducing the concept of "wave trains," and "retarder operators."

The system of waves reflected back on the ground wire due to the arrival at a tower top of a wave $f\left(t^{\prime}\right)$ and the successive reflections

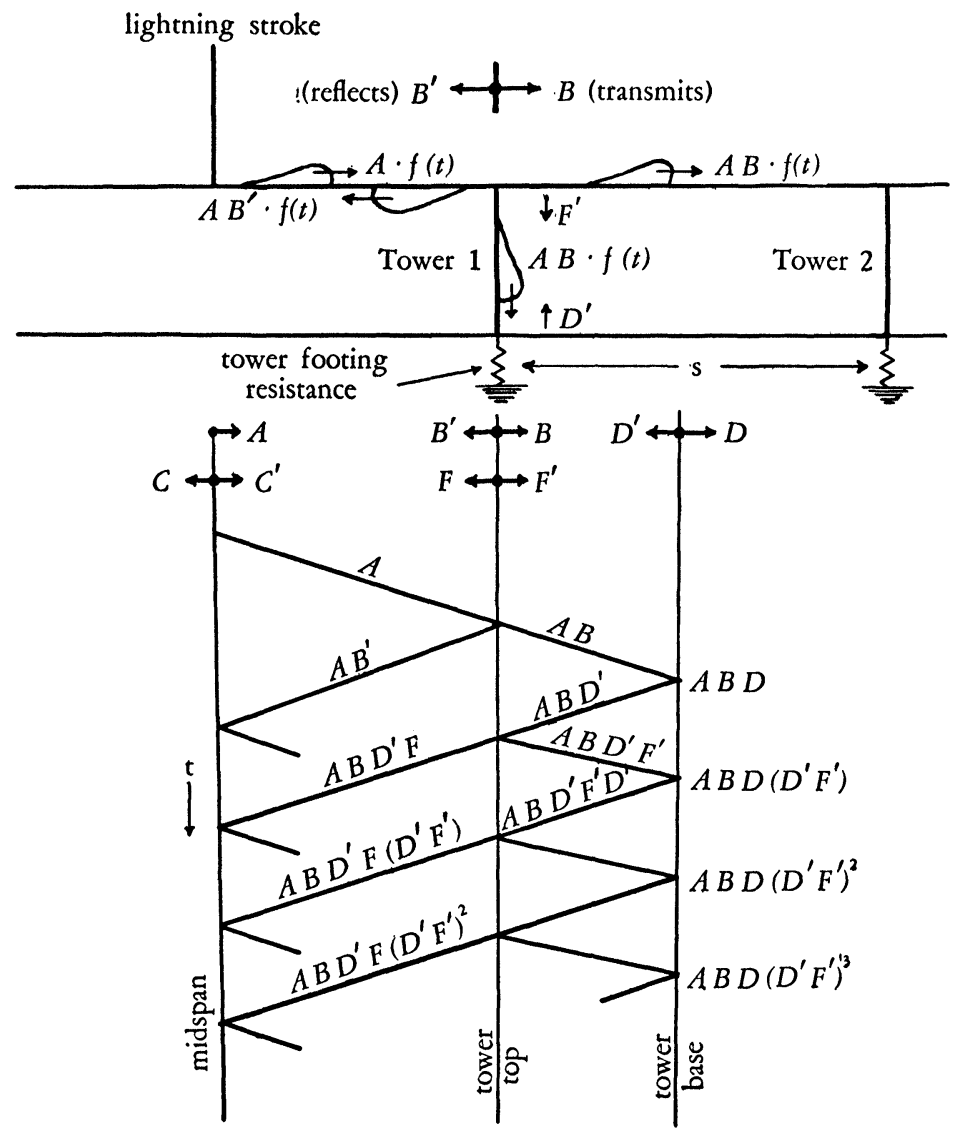

FIG. 3

up and down the tower is seen from the lattice to be a "wave train" of Type I:

$$
\begin{aligned}
e^{\prime} & =B^{\prime} \cdot f\left(t^{\prime}\right)+B D^{\prime} F \cdot f\left(t^{\prime}-2 h\right)+B D^{\prime} F\left(D^{\prime} F^{\prime}\right) \cdot f\left(t^{\prime}-4 h\right)+\cdots \\
& =B^{\prime}\left[f\left(t^{\prime}\right)+\frac{B D^{\prime} F}{B^{\prime}} \sum\left(D^{\prime} F^{\prime}\right)^{m-1} f\left(t^{\prime}-2 h m\right)\right] \\
& =B^{\prime}\left[1+a \sum b^{m-1} \cdot \phi(m)\right] f\left(t^{\prime}\right) \\
& =B^{\prime} \alpha_{m} f(t)
\end{aligned}
$$


in which $\phi(m)$ is a "retarder operator" such that the time of arrival of a wave to which it is applied is retarded by $(2 \mathrm{hm})$, thus

$$
\phi(m) \cdot f\left(t^{\prime}\right)=f\left(t^{\prime}-2 h m\right) .
$$

The wave train transmitted on to the next tower by the incident wave and its tower reflection is the Type II wave train

$$
\begin{aligned}
e^{\prime \prime}=e+e^{\prime} & =B\left[f\left(t^{\prime}\right)+\frac{D^{\prime} F}{B} \sum\left(D^{\prime} F^{\prime}\right)^{m-1} \cdot f\left(t^{\prime}-2 h m\right)\right] \\
& =B\left[1+c \sum b^{m-1} \phi(m)\right] f\left(t^{\prime}\right) \\
& =B \beta_{m} f\left(t^{\prime}\right) .
\end{aligned}
$$

In terms of the wave trains of Types I and II the complete history of the reflections can now be written down.

The initial wave transmitted by a lightning surge to midspan is $A f(t)$ which arrives at the first tower at time $0.5 s$ and gives rise to the Type I wave train of the first order.

$$
e_{1}=A B^{\prime} \alpha_{m} f(t-0.5 s) \text {. }
$$

This wave train arrives at midspan at time $s$ and reflects therefrom as

$$
e_{2}=A B^{\prime} C^{\prime} \alpha_{m} f(t-s) \text {. }
$$

When the reflected wave train arrives at the tower at time (1.5s) it generates a new Type I wave train of the second order

$$
e_{3}=A B^{\prime} C^{\prime} \alpha_{m} \alpha_{n} f(t-1.5 s) \text {. }
$$

Continuing this process we find combinations of the form $\alpha_{m} \alpha_{n} \alpha_{p} \ldots$ in which products are to be interpreted as

$$
\begin{aligned}
\alpha_{m} \alpha_{n} \alpha_{p}= & {\left[1+a \sum b^{m-1} \phi(m)\right]\left[1+a \sum b^{n-1} \phi(n)\right]\left[1+a \sum b^{p-1} \phi(p)\right] } \\
= & {\left[1+3 a \sum b^{m-1} \phi(m)+3 a^{2} \sum \sum b^{m+n-2} \phi(m+n)\right.} \\
& \left.+a^{3} \sum \sum \sum b^{m+n+p-3} \phi(m+n+p)\right] .
\end{aligned}
$$

Now in addition to those wave trains operating between midspan and Tower 1, contributions eventually arrive from neighboring towers. Thus at time $(0.5 s)$ there is transmitted beyond Tower 1 , owing to the initial wave $A f(t)$, the Type II wave train of the first order

$$
E_{1}=A B \beta_{m} f(t-0.5 s) .
$$

This wave train reaches Tower 2 at time $(1.5 s)$ where it generates a Type I wave train of the second order 


$$
E_{2}=A B B^{\prime} \beta_{m} \alpha_{n} f(t-1.5 s) .
$$

This wave train arrives at Tower 1 at time $(2.5 s)$ and generates a third order wave train of Type I

$$
E_{3}=A B^{2} B^{\prime} \beta_{m} \alpha_{n} \beta_{p} f(t-2.5 s),
$$

and this wave train, reaching midspan at $(3 s)$, reflects therefrom as

$$
E_{4}=A B^{2} B^{\prime} C^{\prime} \beta_{m} \alpha_{n} \beta_{p} f(t-3 s) .
$$

With the assistance of the lattice diagram and retarder operators, the potential at any point can be written down. For example, at the top of Tower 1

$$
\begin{aligned}
V= & A B \beta_{m} f(t-0.5 s)+A B B^{\prime} C^{\prime} \alpha_{m} \beta_{n} f(t-1.5 s) \\
& +A B(B C)^{2} \alpha_{m} \alpha_{n} \beta_{p} f(t-2.5 s)+\cdots \\
& +A B^{2} B^{\prime} \beta_{m} \alpha_{n} \beta_{p} f(t-2.5 s)+\cdots \\
& +\cdots
\end{aligned}
$$

Induced lightning surges. Suppose a cloud bearing a charge $Q_{0}$ is over a transmission line, Figure 4 , and is discharging either to

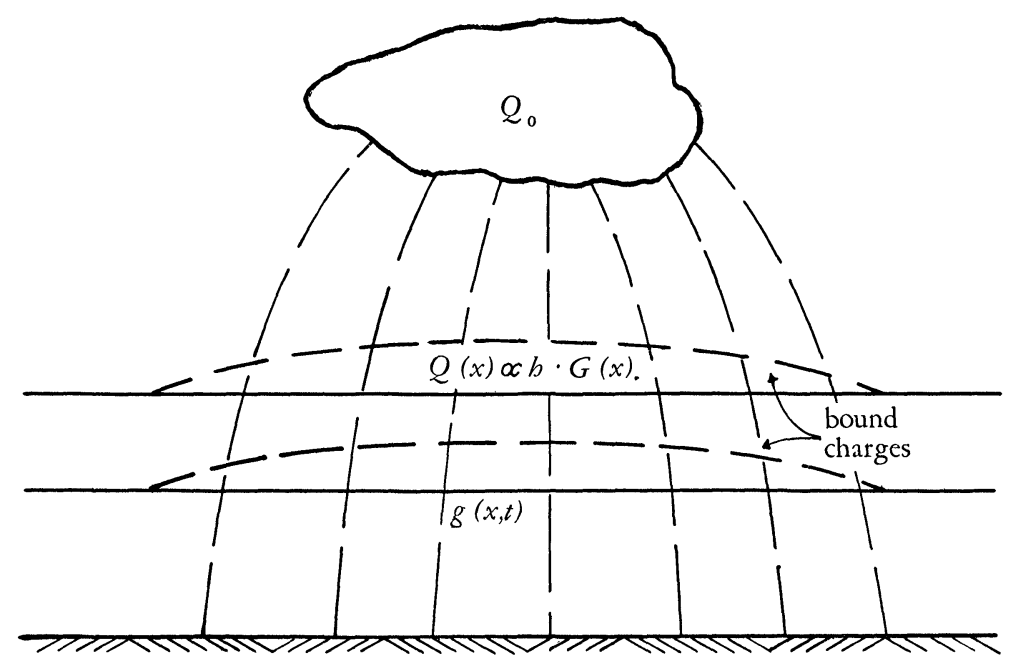

FIG. 4

ground or to another cloud according to some function of time $\psi(t)$; that is, the charge remaining in the cloud at any instant is $Q_{0}[1-\psi(t)]$. Depending upon the shape and size of the cloud charge, its height 
above ground, and its position with respect to the transmission line, the line will experience a gradient

$$
g(x, t)=G(x) \cdot[1-\psi(t)]
$$

in which $G(x)$ represents the initial distribution of gradient (at the beginning of cloud discharge) as a function of distance along the line. Under the influence of this field, charges of opposite sign to that of the cloud will leak over the insulators, or migrate from the remote parts of the system, and collect on the line conductors as bound charges. The density of bound charge at any point $x$ will be proportional to the gradient and to the height $h$ of the conductor above ground (since the field is substantially uniform for a hundred feet or so above ground). These bound charges nullify the potential due to the external field, so that initially the line charges are given by

$$
V_{r}=0=G(x) \cdot h_{r}+p_{r s} Q^{s}, \quad r=1,2, \cdots, n .
$$

Suppose then $m$ of the $n$ wires are ground wires perfectly grounded throughout their length, and let these ideal ground wires be represented by $(j, k)$ indices. The remaining $(n-m)$ wires are power conductors, and will be represented by $(u, v)$ indices.

Now if the field gradient $G(x)$ is suddenly removed, the bound charges on the line wires will not change at the first instant, but those on the ground wires are instantly replaced by new charges $Q^{\prime}$ since the ground wires must remain at zero potential. Therefore

$$
\begin{gathered}
0=p_{j k} Q^{\prime k}+p_{j v} Q^{v}, \\
V_{u}=p_{u k} Q^{\prime k}+p_{u v} Q^{v} .
\end{gathered}
$$

From (2) and (3) all the charges $Q^{s}$ and $Q^{\prime k}$ may be found; and from (4) the potentials $V_{u}$ may be determined. These potentials immediately move out as pairs of traveling waves (in opposite directions). At the first instant, however, the forward and backward waves $f_{u}(x-v t)$ and $F_{u}(x+v t)$ add up to the voltages given by (4), and the resultant current flow must be zero in the isolated power conductors. Thus

$$
\begin{aligned}
f_{u}+F_{u} & =V_{u}, \\
Y^{u v}\left(f_{v}-F_{v}\right) & =0 .
\end{aligned}
$$

Hence

$$
F_{u}=f_{u}=V_{u} / 2,
$$


that is, the forward and backward waves on a conductor are the same shape and magnitude.

However, the release of the bound charge is not instantaneous, but according to the law of cloud discharge $\psi(t)$. The corresponding traveling waves are then given by Duhamel's theorem

(8) $e=\int_{0}^{t}\{f[x+v(t-\tau)]+f[x-v(t-\tau)]\} \frac{\partial \psi(\tau)}{\partial \tau} d \tau$
(9) $=\lim _{\Delta t \rightarrow 0} \sum_{0}^{n}\{f[x+v(n-k) \Delta t]+f[x-v(n-k) \Delta t]\} \Delta \psi_{k}$

where $n \cdot \Delta t=t, k \cdot \Delta t=\tau$ and $\Delta \psi_{k}=\psi[(k+1) \Delta t]-\psi[k \cdot \Delta t]$. The application of the integral is limited to relatively simple expressions for $f$ and $\psi$, but the summation can be used for any functions whose graphs are known or assumed. Ultimately, since both $f$ and $\psi$ derive from experimental data, it is best to use the summation expression. Both graphical and tabular methods have been devised for its ready application, and engineering solutions are quickly arrived at.

Equation (8) can also be derived by setting up the conditions in terms of retarded potentials. Very few engineers deal with retarded potentials, whereas a considerable number of them are familiar with Duhamel's theorem through Heaviside's operational calculus.

LEHIGH UNIVERSITY 